\title{
Intrinsic Properties of Single Graphene Nanoribbons in Solution: Synthetic and Spectroscopic Studies
}

Yinjuan Huang ${ }^{1 \#}$, Fugui Xu ${ }^{1 \#}$, Lucia Ganzer ${ }^{2}$, Franco V. A. Camargo ${ }^{2}$, Tetsuhiko Nagahara ${ }^{2,3}$, Joan Teyssandier $^{4}$, Hans van Gorp ${ }^{4}$, Kristoffer Basse ${ }^{5}$, Lasse Arnt Straasø ${ }^{5}$, Vaiva Nagyte ${ }^{6}$, Cinzia Casiraghi $^{6}$, Michael Ryan Hansen, Steven De Feyter ${ }^{4}$, Deyue Yan ${ }^{1}, K_{\text {Klaus Müllen }}^{8}$, Xinliang Feng Giulio Cerullo ${ }^{2 *}$, and Yiyong $\mathrm{Mai}^{1 *}$

${ }^{1}$ School of Chemistry and Chemical Engineering, Shanghai Jiao Tong University, 8oo Dongchuan RD, Shanghai 200240, China; ${ }^{2}$ IFN-CNR, Dipartimento di Fisica, Piazza L. da Vinci 32, 20133 Milano, Italy; 3 Department of Chemistry and Materials Technology, Kyoto Institute of Technology, 6o6-8585 Kyoto, Japan; 4 Division of Molecular Imaging and Photonics, Department of Chemistry, KU Leuven Celestijnenlaan, 200 F, B-30o1 Leuven, Belgium; ${ }^{5}$ Interdisciplinary Nanoscience Center, Aarhus University, Gustav Wieds Vej 14, DK-80oo Aarhus C, Denmark; ${ }^{6}$ School of Chemistry, University of Manchester, Oxford Road, Manchester M139PL, United Kingdom; 7 Institute of Physical Chemistry, Westfälische Wilhelms-Universität Münster, Corrensstr. 28/30, D-48149 Münster, Germany; ${ }^{8}$ Max Planck Institute for Polymer Research, Ackermannweg 10, 55128, Mainz, Germany; ${ }^{9}$ Department of Chemistry and Food Chemistry, Technische Universität Dresden, Mommsenstrasse 4, 01062 Dresden, Germany.

Supporting Information

ABSTRACT: Structurally defined graphene nanoribbons (GNRs) are of interest due to their unique optical and electronic properties. However, strong $\pi-\pi$ interactions typically cause distinct aggregation in liquid phase, thus hampering their solution processability. Here, we report a novel type of structurally defined GNRs with uniform width of $1.7 \mathrm{~nm}$ and average length up to $58 \mathrm{~nm}$. These GNRs are decorated with pending Diels-Alder cycloadducts of anthracenyl units and $\mathrm{N}$ n-hexadecyl maleimide. The resultant bulky side groups on GNRs afford excellent dispersibility with concentrations of up to $5 \mathrm{mg} \mathrm{mL}^{-1}$ in many organic solvents such as tetrahydrofuran (THF), two orders of magnitude higher than the previously reported GNRs. Multiple spectroscopic studies confirm that dilute dispersions in THF ( $\left.<0.1 \mathrm{mg} \mathrm{mL}^{-1}\right)$ consist mainly of nonaggregated ribbons, exhibiting near-infrared emission, high quantum yield $(\sim 9.1 \%)$ and long lifetime $(\sim 8.7 \mathrm{~ns})$ of photoluminescence. This unprecedented dispersibility allows resolving in real-time ultrafast excited-state dynamics of the GNRs, which displays features of small isolated molecules in solution. GNRs. This study achieves a breakthrough in the dispersion of GNRs, which opens the door for unveiling obstructed GNR based physical properties and potential applications.

Structurally defined graphene nanoribbons (GNRs) have attracted increasing interest due to their tunable optical, electronic and magnetic properties by tailoring their width and/or edge structures. ${ }^{1-18}$ Two "bottom-up" strategies, including surface-assisted ${ }^{1-7}$ and solution-based organic synthesis, ${ }^{9-17}$ were developed for GNRs. In contrast to the surface-mediated method, the solution synthesis shows significant advantages in large-scale preparation of liquid-phase-dispersible GNRs.9${ }_{17}$ However, controlling the physical properties of GNRs, such as optical bandgap and carrier mobility, will be only possible if individual GNRs can be achieved. Otherwise aggregation effects, which could so far never be excluded, would obscure their intrinsic physical properties..$^{10-17}$

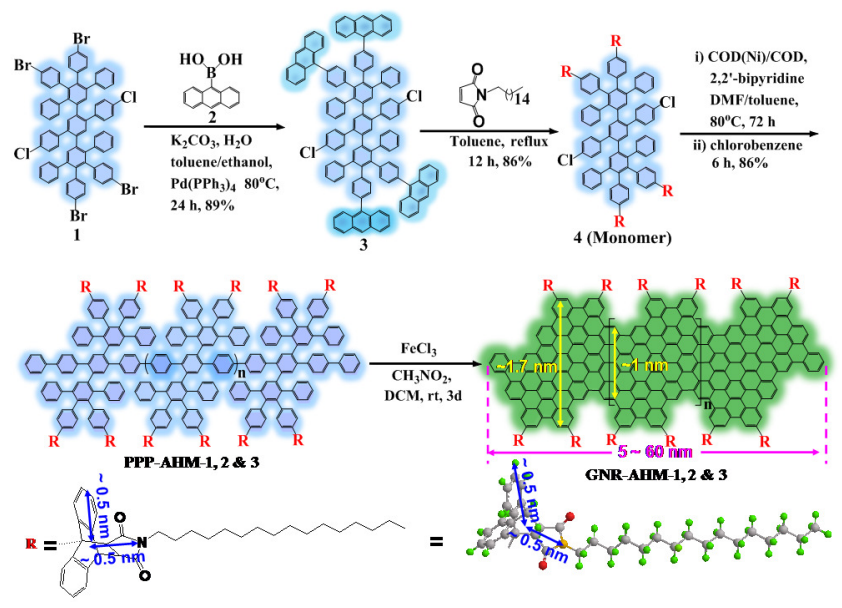

Scheme 1. Synthesis of GNR-AHM. The bulky AHM group size is determined by Chem3D. GNR-AHM-1, 2 \& 3 possess average lengths of ca. 6,11 and $58 \mathrm{~nm}$, respectively.

Here, we demonstrate the solution synthesis of a new type of GNRs (hereafter GNR-AHM) with a uniform width of $1.7 \mathrm{~nm}$ and average lengths up to $58 \mathrm{~nm}$, which are decorated with pending Diels-Alder cycloadducts of anthracenyl units and $\mathrm{N}$ n-hexadecyl maleimide (AHM) (Scheme 1). The AHM side groups have radius of $\sim 0.5 \mathrm{~nm}$, which is larger than the interlayer spacing of graphite $(\sim 0.34 \mathrm{~nm}),{ }^{19-21}$ effectively hindering the $\pi-\pi$ stacking (Scheme $\mathrm{S}_{1}$ ). GNR-AHMs show unprecedented dispersibility in many organic solvents (e.g. tetrahydrofuran, THF) with concentrations of up to $5 \mathrm{mg} \mathrm{mL}^{-1}$ (for GNR backbone excluding AHM unless otherwise mentioned). 
Atomic force microscopy (AFM) analysis of GNR-AHM deposited on graphite substrate shows a periodic self-assembled monolayer structure with a lateral side-by-side alignment of single ribbons. Dynamic light scattering (DLS), steady state and transient absorption (TA) spectroscopies suggest a singleribbon feature of the GNRs in dilute dispersions (e.g. $<0.1 \mathrm{mg}$ $\mathrm{mL}^{-1}$ in THF), thus allowing to unravel the photophysical properties of isolated GNRs in liquid phase.

The chemical synthesis of GNR-AHM is illustrated in Scheme 1 (see Supporting Information -SI- for details). First, a tetraanthracenyl and dichloro-substituted oligophenylene $\mathbf{3}$ was synthesized by the Suzuki reaction of tetrabromo- and dichloro-substituted oligophenylene $\mathbf{1}^{17,22}$ and 9-anthraceneboronic acid $\mathbf{2}$, in $89 \%$ yield. Compound $\mathbf{3}$ was further reacted with $N$-n-hexadecyl maleimide through a Diels-Alder cycloaddition, yielding a dichloro-substituted oligophenylene monomer 4 with four AHM edge groups (86\% yield). The successful synthesis of $\mathbf{3}$ and $\mathbf{4}$ was demonstrated by ${ }^{1} \mathrm{H}$ and ${ }^{13} \mathrm{C}$ nuclear magnetic resonance (NMR), matrix-assisted laser desorption/ionization time-of-flight (MALDI-TOF) mass, and elemental analyses (Figures $\mathrm{S}_{2}-\mathrm{S}_{7}$ and Pages $\mathrm{S}_{7}-\mathrm{S}_{11}$ ). Second, laterally expanded poly-para-phenylene precursor with AHM edge substituents (PPP-AHM) was synthesized by AA-type Yamamoto polymerization of monomer 4. Recycling gel permeation chromatography (GPC) yielded three PPP-AHM samples (PPP-AHM-1, 2 \& 3) with number-average molecular weights $\left(M_{\mathrm{n}} \mathrm{s}\right)$ ranging from 13,000 to 124,000 and polydispersity indices (PDI) of 1.2-1.5 (determined by GPC, see Figure $\mathrm{S} 12)$. Finally, through intramolecular oxidative cyclodehydrogenation in $\mathrm{CH}_{2} \mathrm{Cl}_{2}$ using $\mathrm{FeCl}_{3}$ as the Lewis acid and oxidant, PPP-AHMs were converted into arm-chair edged GNR-AHMs with uniform width $(1.7 \mathrm{~nm})$ and different lengths. Since PPPAHM-1, $2 \& 3$ possess 4,8 and 42 repeating units $(1.38 \mathrm{~nm}$ for each unit), calculated from their $M_{\mathrm{n}} \mathrm{s}$, the corresponding average lengths of GNR-AHM-1, $2 \& 3$ are 6,11 and $58 \mathrm{~nm}$, respectively.

Fourier transform infrared (FTIR) spectra demonstrate the efficient "graphitization" and planarization of PPP into GNR (Figures $\mathrm{S}_{14}-\mathrm{S}_{16}$ ). In the spectra of GNR-AHMs the signals attributable to the aromatic $\mathrm{C}-\mathrm{H}$ stretching vibrations and the out-of-plane (opla) $\mathrm{C}-\mathrm{H}$ deformation are significantly attenuated compared with those of PPP-AHMs, while typical opla bands for aromatic $\mathrm{C}-\mathrm{H}$ at the armchair edge of the GNR basal plane appear. ${ }^{13,16,23}$ Solid-state NMR analyses prove that the semiflexible polyphenylene precursor becomes rigid and planar after "graphitization" (Figure S18).13,16,17 Particularly, the 2D ${ }^{1} \mathrm{H}-{ }^{1} \mathrm{H}$ double quantum-single quantum (DQ-SQ) correlation spectra of PPPs exhibit narrow and resolved signals whereas those of GNRs show broad, stretched, and split ridge signals in the aromatic region (Figures S18b,e). Moreover, the 2D DQSQ spectra display cross peaks that originate from the close spatial proximity of the two types of aromatic protons at the edge of the GNRs (Figure S18e), confirming the efficient planarization. The almost unchanged ${ }^{13} \mathrm{C}\left\{{ }^{1} \mathrm{H}\right\} \mathrm{CP} / \mathrm{MAS}$ NMR spectra indicate that the AHM groups are unaffected by the cyclodehydrogenation reaction (Figure S18c,f).

The first-order Raman spectra of the resulting GNRs exhibit typical D and $G$ peaks (Figure 1a), as observed in other reported bottom-up synthesized GNRs.13,16 The D peak is not activated by defects, which is similar to what is observed in aromatic molecules, where the atomic vibrations of the $D$ peak are Raman active and do not result from defects. ${ }^{24}$ In the lowfrequency region, GNRs exhibit a characteristic peak at $\sim 258$ $\mathrm{cm}^{-1}$, which is associated to the radial breathing-like layer mode (RBLM). ${ }^{25}$ This is a fingerprint of narrow $(<2 \mathrm{~nm})$ and atomically precise GNRs. ${ }^{26}$ The RBLM peak is more intense at $638 \mathrm{~nm}$ than $532 \mathrm{~nm}$ excitation, due to the electronic resonance. ${ }^{25,26}$ By using the equation $w=3222 / v_{\text {RBLM }} \AA$, where $w$ is the width and $v_{\mathrm{RBLM}}$ is the RBLM wavenumber, the estimated width is $\sim 1.3 \mathrm{~nm}$, in reasonable agreement with the structurally determined value (1.7 $\mathrm{nm}$, Scheme 1$)$.

Thanks to the bulky AHM, GNR-AHMs show superior dispersibility in organic solvents including THF, dichloromethane (DCM), toluene, and chlorobenzene (CB), among others (Figure $\mathrm{lb}$ ). For example, mild shaking or sonication of GNR-AHM in these solvents generates black homogeneous dispersions with concentrations of up to $5 \mathrm{mg} \mathrm{mL}^{-1}$ (Figures S20-S21). The dispersions were stable for over three months without precipitation. Such a dispersibility greatly surpasses those reported for alkylated GNRs (<0.05 $\mathrm{mg} \mathrm{mL}^{-1}$ in THF), which require strong sonication for hours and are stable for several days at most. 13,16,17 In addition, DLS analyses give similar unimodal narrow size distributions with average hydrodynamic diameters $\left(D_{\mathrm{h}}\right)$ of $40 \sim 60 \mathrm{~nm}$ for GNR-AHM-3 in the above-mentioned solvents with concentrations from 0.01 to $0.1 \mathrm{mg} \mathrm{mL}^{-1}$ (Figure $\mathrm{S}_{23}$ ). $D_{\mathrm{h}}$ being close to the length $(\sim 6 \mathrm{omm})$ of individual GNR-AHM-3 and not increasing with increasing concentration (o.01 0.1 $\mathrm{mg} \mathrm{mL}^{-1}$ ) suggests a single-ribbon character or very weak aggregation of the GNRs. Furthermore, GNR-AHMs can also be dispersed, although with more aggregation, in some polar solvents including $N, N$-dimethylformamide, dimethylsulfoxide, acetone and ethyl acetate, etc. (Fig-

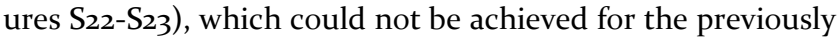
reported alkyl substituted or polymer functionalized GNRs. ${ }^{13,16,17}$
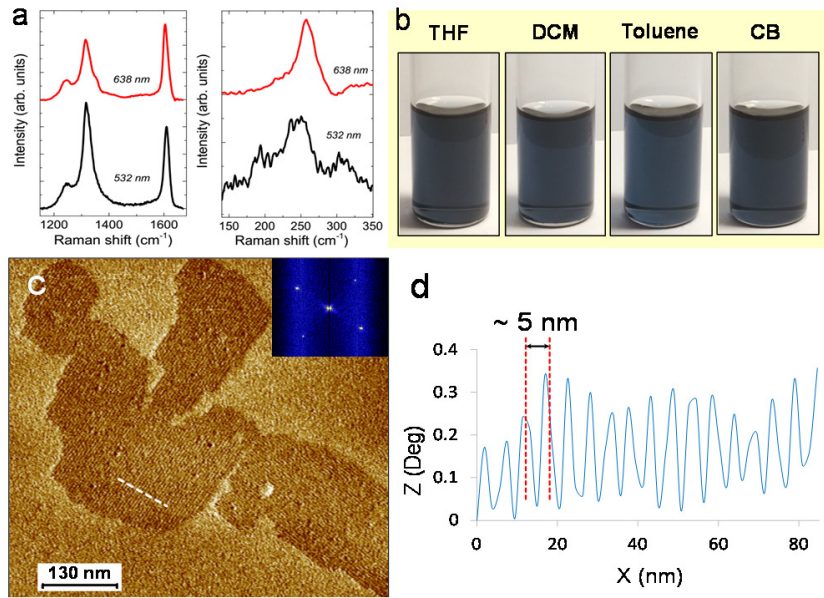

Figure 1. (a) Raman spectra of GNR-AHM-3, excited at 532 and 638 nm. (b) Digital photographs of GNR-AHM-3 in various solvents (o.1 $\mathrm{mg} \mathrm{mL} \mathrm{m}^{-1}, 5 \mathrm{mg} \mathrm{mL}^{-1}$ dispersions are shown in Figure S21). (c) AFM phase image of self-assembled domains of the GNRs on HOPG, with the corresponding fast Fourier transform (FFT) shown in inset. Each set of two points (one point and its central inversion) in the FFT corresponds to a periodicity of $\sim 5 \mathrm{~nm}$ along one direction. The presence of two pairs of points comes from the existence of two domains with lamellar periodicity in two different directions. (d) Line profile along the white dotted line in panel (c).

Such excellent dispersibility allows drop-casting diluted dispersions of GNR-AHMs in 1,2,4-trichlorobenzene (TCB) on highly oriented pyrolytic graphite (HOPG). Self-assembled lamellae were imaged by AFM after solvent evaporation (Figures 1c and S24-S25). The domain sizes vary roughly from 10,0oo to 
250,000 $\mathrm{nm}^{2}$, depending on the GNR concentration. AFM topographic images reveal a uniform thickness of $\sim 0.4 \mathrm{~nm}$ for the lamellae (Figure S25), demonstrating their monolayer feature. The self-assembled monolayers exhibit the same internal lateral periodicity of $5.0 \pm 0.4 \mathrm{~nm}$ (Figure $1 \mathrm{~d}$ ), which is compatible with the width of a single GNR-AHM. The perfect lamellar order as well as the uniformity in periodicity of the monolayers demonstrates the homogeneity in shape and width of the GNRs. Moreover, the longitudinal sizes (45 500 $\mathrm{nm}$ ) of the lamellae are larger than the length of single GNRs, indicating an end-to-end alignment fashion of the GNRs in addition to lateral side-by-side arrangement. The fact that the GNRs appeared as organized lamellae is most likely due to van der Waals interactions among the alkyl side chains. Contrary to what happens in solution, the AHM groups do not appear to significantly affect the self-assembly behavior on the surface. Their effect is nevertheless detectable in the preparation of the films, since organized GNR-AHM monolayers were formed without any ultrasonication, as opposed to reported alkyl substituted GNRs that required an additional step of strong ultrasonication prior to deposition on HOPG.13,16,17

The absorption spectra of different lengths in THF are very similar and contrast with those for the previously reported poly(ethylene oxide) (PEO)-functionalized GNRs (GNR-PEO) (Figure 2a). ${ }^{17}$ While GNR-PEO shows featureless absorption in the visible with a long tail extending to the near-infrared (NIR) due to scattering, a typical sign of aggregation, GNR-AHMs have intense absorption limited to the region below $750 \mathrm{~nm}$. There is almost perfect agreement between the absorption and PL (Figure 2b) of GNR-AHM-1 and 3, both of which are red-shifted compared to the monomer (Figure Sil). Thus, the exciton is delocalized over no more than four monomer units for this edge-structure. A linear relationship between PL intensity and concentration $\left(<0.1 \mathrm{mg} \mathrm{mL}^{-1}\right)$ further confirms the single-ribbon character (Figure S26). Further, GNR-AHM-3 exhibits a 9.1\% PL quantum yield as well as a lifetime of $8.7 \mathrm{~ns}$ (Table $\mathrm{S} 1$ ); these values could not be measured for other reported structurally-defined GNRs due to aggregation-induced PL quenching.

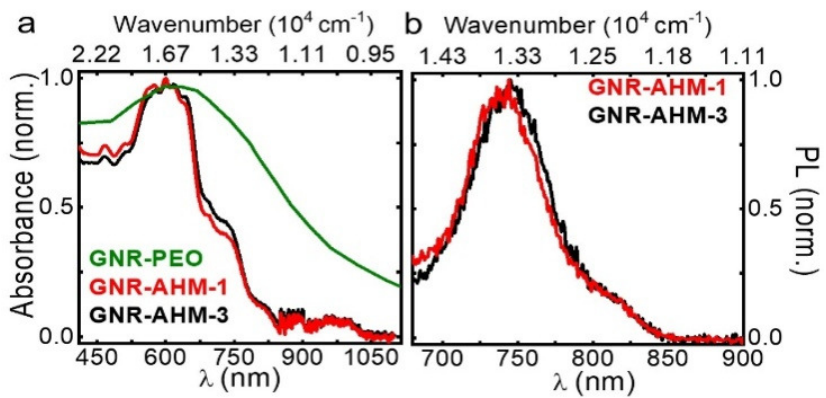

Figure 2. Absorption (a) and PL (b) spectra of GNR-AHMs in THF (o.03 and $0.003 \mathrm{mg} \mathrm{mL}^{-1}$ for absorption and PL measurements, respectively). GNR-PEO spectrum is reproduced from Ref. 17.

Ultrafast TA experiments using $100 \mathrm{fs}, 730 \mathrm{~nm}$ pump pulses were performed on GNR-AHM-1 \& 3 giving similar results. Figure za summarizes the TA spectra of GNR-AHM-3 (see Figure S28 for GNR-AHM-1). Unlike the absorption (Figure 2a), the TA spectra are remarkably simple, consisting of three positive peaks at 738, 66o and $615 \mathrm{~nm}$. The peaks at 660 and $615 \mathrm{~nm}$ contain only ground state bleach (GSB) features, while the peak at $738 \mathrm{~nm}$ includes a stimulated emission (SE). The separation between those peaks (Figure $3 a$ ) matches the $G$ and $2 \mathrm{D}$ peaks in the Raman spectrum (Figure 1a). At early delay times we observe a positive peak around $820 \mathrm{~nm}$ corresponding to SE from the lowest energy exciton to $G$ and $D$ vibrational replicas of the ground stat. Therefore, even the $58 \mathrm{~nm}$ long GNRAHM-3 behaves like a small organic molecule in solution, with the electronic structure of a vibronic progression.

Typical TA dynamics timetraces (Figure 3b) show an important 250 fs decay at 738 and $820 \mathrm{~nm}$ (containing SE signals) which is only weakly present at the other bands (see SI for global fit). ${ }^{27}$ This component is assigned to exciton migration to the few remaining aggregate states found in the GNR solution. Two slower decay components of 9 and 290 ps are present equally in all bands, and are similar to reported decays on small graphene molecules and carbon nanotubes. ${ }^{28}$ As expected for molecules of this size, no rotational reorientation was observed. ${ }^{29}$

Finally, the nature of the lowest electronic transition is investigated with $2 \mathrm{D}$ electronic spectroscopy (2DES) using $\sim 10-$ fs NIR pulses. ${ }^{30}$ 2DES yields a correlation map between pump and probe wavelengths ( $\left.\lambda_{\text {pump }}, \lambda_{\text {probe }}\right)$ for each waiting time $t_{2}$ between them..$^{31}$ Figure $3 \mathrm{c}$ displays 2 DES maps of GNR-AHM3 at $t_{2}=50,200$ and $1500 \mathrm{fs}$; the main feature is a peak stretched along the diagonal $\left(\lambda_{\text {pump }}=\lambda_{\text {probe }}\right)$ with a cross peak parallel to it below the diagonal. Their separation of $\sim 1600 \mathrm{~cm}^{-1}$ shows that the diagonal peak consists of two bands, with the high energy one being a vibronic replica. The diagonal elongation marks an inhomogeneously broadened transition, implying that a distribution of microscopic environments shifts individual transitions. As the waiting time increases, molecules usually stochastically probe the different environments, leading to spectral diffusion and eventually resulting in a round lineshape. ${ }^{22}$ However, the main peak remains strongly stretched even at 1.5 ps and TA spectra show no major broadening up to 900 ps (Figure $S_{32}$ ). This suggests a rigid but diverse range of microscopic structures around the GNRs, which could be related to the AHM side groups.

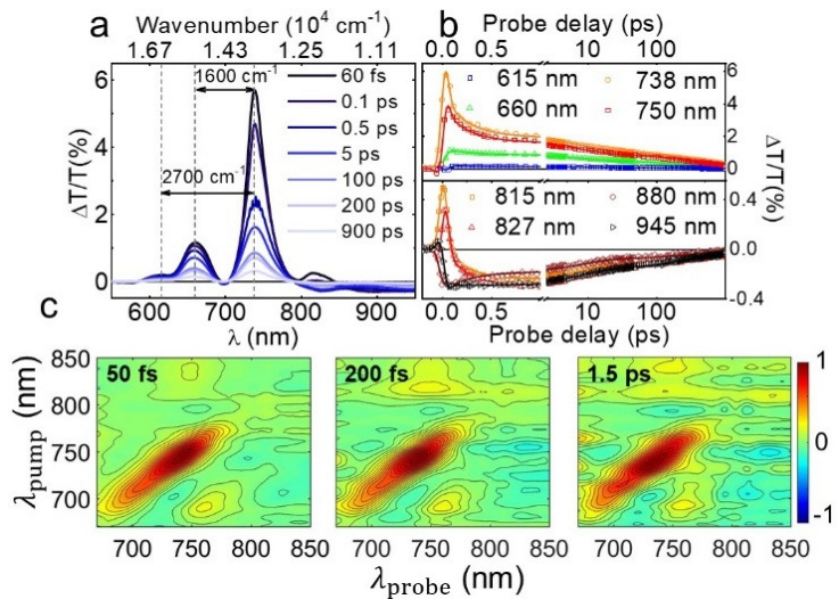

Figure 3. (a) TA spectra of GNR-AHM-3 $\left(0.5 \mathrm{mg} \mathrm{mL}^{-1}\right)$ for different delays; (b) TA at specific wavelengths (symbols) with corresponding fits (solid lines). (c) Normalized NIR 2 DES maps $\left(2 \mathrm{mg} \mathrm{mL}^{-1}\right)$ at $\mathrm{t}_{2}=50$, 200 and 1500 fs.

In summary, we report grafting of bulky side groups at the edge of structurally defined GNRs, affording breakthrough in dispersion of single GNRs. This allowed us to study the electronic structure and optical properties of single ribbons. As synthetic methods towards GNRs with other edge structures were achieved, this approach can again be used to dissolve in- 
dividual ribbons of other architectures, such as cove- or partially zigzag-edged ones. Control over the electronic and optical properties of GNRs with a single-ribbon feature holds promise in many potential applications, including field-effect transistor devices, light harvesting, fluorescence imaging, and photothermal conversion.

\section{ASSOCIATED CONTENT}

\section{Supporting Information}

Experiments details, supporting figures and calculations, etc.

\section{AUTHOR INFORMATION}

\section{Corresponding Authors}

* mai@sjtu.edu.cn (Y. M.); giulio.cerullo@fisi.polimi.it (G. C.)

\# Contributed equally.

\section{Notes}

The authors declare no competing financial interest.

\section{ACKNOWLEDGMENTS}

The authors thank the financial support from National Natural Science Foundation of China (21320102006, 21774076, 51573091 and 91527304), Program of the Shanghai Committee of Science and Technology (17JC1403200 and 16JC1400703), EC under Graphene Flagship (CNECT-ICT-604391 and 785219 GrapheneCore 2), ERC under the European Union's Horizon 2020 research and innovation program (648417), EPSRC in the framework of the CDT Graphene NOWNANO and the National Physical Laboratory of London.

\section{REFERENCES}

(1) Nguyen, G. D.; Tsai, H.-Z.; Omrani, A. A.; Marangoni, T.; Wu, M.; Rizzo, D. J.; Rodgers, G. F.; R Cloke,. R. R.; Durr, A.; Sakai, Y.; Liou, F.; Aikawa, A. S.; Chelikowsky, J. R.; Louie, S. G.; Fischer, F. R.; Crommie, M. F. Nat. Nanotech. 2017, 12, 1077.

(2) Cai, J.; Ruffieux, P.; Jaafar, R.; Bieri, M.; Braun, T.; Blankenburg, S.; Muoth, M.; Seitsonen, A. P.; Saleh, M.; Feng, X.; Müllen, K.; Fasel, R. Nature 2010, 466, 470.

(3) Cai, J.; Pignedoli, C. A.; Feng, X.; Müllen, K.; Fasel, R. Nat. Nanotech. 2014, 9, 896.

(4) Chen, Y. C.; Cao, T.; Chen, C.; Pedramrazi, Z.; Haberer, D.; Oteyza, D. G.; Fischer, F. R.; Louie, S. G.; Crommie, M. F. Nat. Nanotech. 2o15, $10,156$.

(5) Kawai, S.; Saito, S.; Osumi, S.; Yamaguchi, S.; Foster, A. S.; Spijker, P.; Meyer, E. Nat. Commun. 2015, 6, 8098.

(6) Ruffieux, P.; Wang, S.; Yang, B.; Sánchez-Sánchez, C.; Liu, J.; Dienel, T.; Talirz, L.; Shinde, P.; Pignedoli, C. A.; Passerone, D.; Dumslaff, T.; Feng, X.; Müllen, K.; Fasel, R. Nature 2016, 531, 489.

(7) Wang, S.; Talirz, L.; Pignedoli, C. A.; Feng, X.; Müllen, K.; Fasel, R.; Ruffieux, P. Nat. Commun. 2016, 7, 11507.

(8) Jordan, R. S.; Li, Y. L.; Lin, C.; McCurdy, R. D.; Lin, J. B.; Brosmer, J. L.; Marsh, K. L.; Khan, S. I.; Houk, K. N.; Kaner, R. B.; Rubin, Y. J. Am. Chem. Soc. 2017, 139, 15878.

(9) Yang, X.; Dou, X.; Rouhanipour, A.; Zhi, L.; Müllen, K. J. Am. Chem. Soc. 2008, 130, 4216.

(10) (a) Keerthi, A.; Radha, B.; Rizzo, D.; Lu, H.; Cabanes, V. D.; Hou, I. C.-Y.; Beljonne, D.; Cornil, J.; Casiraghi, C.; Baumgarten, M.; Müllen, K.; Narita, A. J. Am. Chem. Soc., 2017, 139, 16454. (b) Jänsch,
D.; Ivanov, I.; Zagranyarski, Y.; Duznovic, I.; Baumgarten, M.; Turchinovich, D.; Li, C.; Bonn, M.; Müllen, K. Chem. Eur. J. 2017, 23, 4870 .

(11) Schwab, M. G.; Narita, A.; Hernandez, Y.; Balandina, T.; Mali, K. S.; Feyter, S. D.; Feng, X.; Müllen, K. J. Am. Chem. Soc. 2012, 134, 18169. (12) Dçssel, L.; Gherghel, L.; Feng, X.; Müllen, K.; Angew. Chem. Int. Ed. 2011, 50, 2540.

(13) Narita, A.; Verzhbitskiy, I. A.; Frederickx, W.; Mali, K. S.; Jensen, S. A.; Hansen, M. R.; Bonn, M.; Feyter, S. D.; Casiraghi, C.; Feng, X.; Müllen, K. ACS Nano 2014, 8, 11622.

(14) Gao, J.; Uribe-Romo, F. J.; Saathoff, J. D.; Arslan, H.; Crick, C. R.; Hein, S. J.; Itin, B.; Clancy, P.; Dichtel, W. R.; Loo, Y. L. ACS Nano 2016, $10,4847$.

(15) Yang, W.; Lucotti, A.; Tommasini, M.; Chalifoux, W. A. J. Am. Chem. Soc. 2016, 138, 9137.

(16) Narita, A.; Feng, X.; Hernandez, Y.; Jensen, S. A.; Bonn, M.; Yang, H.; Verzhbitskiy, I. A.; Casiraghi, C.; Hansen, M. R.; Koch, A. H. R.; Fytas, G.; Ivasenko, O.; Li, B.; Mali, K. S.; Balandina, T.; Mahesh, S.; Feyter, S. D.; Müllen, K. Nat. Chem. 2013, 6, 126.

(17) Huang, Y.; Mai, Y.; Beser, U.; Teyssandier, J.; Velpula, G.; Gorp, H.; Hansen, L. A.; Hansen, M. R.; Rizzo, D.; Casiraghi, C.; Yang, R.; Zhang, G.; Wu, D.; Zhang, F.; Yan, D.; Feyter, S. D.; Mullen, K.; Feng, X. J. Am. Chem. Soc. 2016, 138, 10136.

(18) (a) M. Ezawa, Phys. Rev. B. 20o6, 73, 045432. (b) Ivanov, I.; Yunbin, H.; Osella, S.; Beser, U.; Wang, H. I.; Beljone, D.; Narita, A.; Müllen, K.; Turchinovich, D.; Bonn, M. J. Am. Chem. Soc. 2017, 139, 7982. (19) Huang, Y.; Dou, W.-T.; Xu, F.; Ru, H.-B.; Gong, Q.; Wu, D.; Yan, D.; Tian, H.; He, X.-P.; Mai, Y.; Feng. X. Angew. Chem. Int. Ed. 2018, $57,3366$.

(20) Xu, Y.; Bai, H.; Lu, G.; Li, C.; Shi. G. J. Am. Chem. Soc. 20o8, 130, 5856.

(21) Ramanathan, T.; Abdala, A. A.; Stankovich, S.; Dikin, D. A.; Herrera-Alonso, M.; Piner, R. D.; Adamson, D. H.; Schniepp, H. C.; Chen, X.; Ruoff, R. S.; Nguyen, S. T.; Aksay, I. A.; Prud'homme, R. K.; Brinson. L. C. Nat. Nanotech. 2008, 3, 327.

(22) (a) Huang, Y.; Mai, Y.; Yang, X.; Beser, U.; Liu, J.; Zhang, F.; Yan, D.; Müllen, K.; Feng, X. J. Am. Chem. Soc. 2015, 137, 11602. (b) Huang, Y.; Yuan, R.; Xu, F.; Mai, Y.; Feng, X.; Yan, D. Polym. Chem. 2016, 7, 1234.

(23) Centrone, A.; Brambilla, L.; Renouard, T.; Gherghel, L.; Mathis, C.; Müllen, K.; Zerbi, G. Carbon 2005, 43, 1593.

(24) Castiglioni, C.; Tommasini, M.; Zerbi. G. Philosophical Phil. Trans. R. Soc. Lond. A 2004, 362, 2425.

(25) Zhou, J.; Dong. J. Appl. Phys. Lett. 2007, 91, 173108.

(26) Verzhbitskiy, I. A.; Corato, M. D.; Ruini, A.; Molinari, E.; Narita, A.; Hu, Y.; Schwab, M. G.; Bruna, M.; Yoon, D.; Milana, S.; Feng, X.; Müllen, K.; Ferrari, A. C.; Casiraghi, C.; Prezzi, D. Nano Lett., 2016, 16, 3442.

(27) Snellenburg, J. J.; Laptenok, S. P.; Seger, R.; Müllen, K. M.; van Stokkum, I. H. M. J. Stat. Softw., 2012, 49, 1.

(28) (a) Soavi, G.; Dal Conte, S.; Manzoni, C.; Viola, D.; Narita, A; Hu, Y.; Feng, X.; Hohenester, U.; Molinari, E.; Prezzi, D.; Müllen, K.; Cerullo, G. Nat. Commun., 2017, 7, 11010. (b) Paternò, G. M.; Chen, Q.; Wang, X.-Y.; Liu, J.; Motti, S. G.; Petrozza, A.; Feng, X.; Lanzani, G.; Müllen, K.; Narita, A.; Scotognella, F. Angew. Chem. Int. Ed. 2017, 56, 6753.

(29) Camargo, F. V. A.; Anderson, H. L.; Meech, S. R.; Heisler, I. A. J. Phys. Chem. B 2015, 119, 14660.

(30) Maiuri, M.; Réhault, J.; Carey, A.-M.; Hacking, K.; Garavelli, M.; Lüer, L.; Polli, D.; Cogdell, R.J.; Cerullo, G. J. Chem. Phy. 2015, 142, 212433.

(31) (a) Jonas, D. M. Annu. Rev. Phys. Chem. 2003, 54, 425; (b) Nuernberger, P.; Ruetzel, S.; Brixner, T. Angew. Chem. Int. Ed. 2015, 54, 11368. (32) (a) Šanda, F.; Perlík, V.; Lincoln, C. N.; Hauer, J. J. Phys. Chem. A 2015, 119, 10893; (b) Moca, R.; Meech, S.R.; Heisler, I.A. J. Phys. Chem. $B$ 2015, 119, 8623. 
Intrinsic Properties of Single Graphene Nanoribbons in Solution: Synthetic and Spectroscopic Studies

Graphic for Table of Content

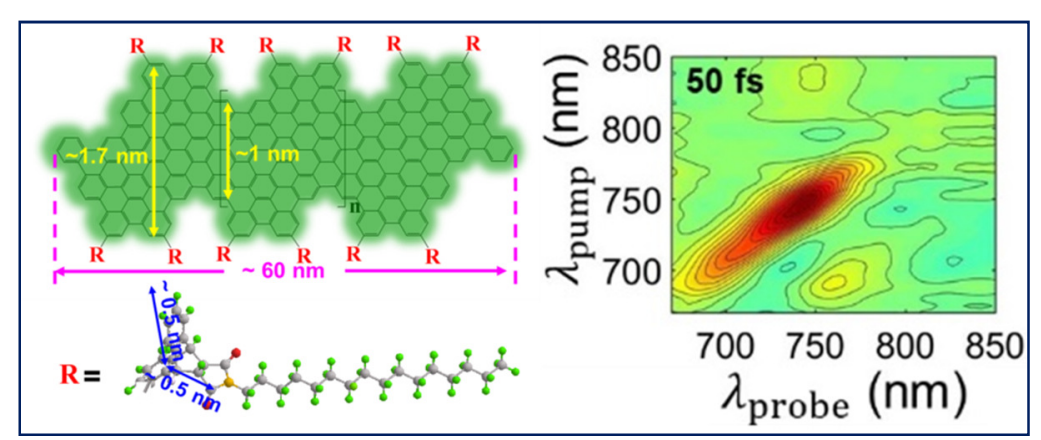

